\title{
Ontology-Based Distributed Health Record Management System
}

\author{
Calin Cenan ${ }^{1}$, Gheorghe Sebestyen ${ }^{1}$, Gavril Saplacan ${ }^{1}$, Dan Radulescu ${ }^{2}$ \\ ${ }^{1}$ Dept. of Computer Science, Technical University of Cluj Napoca, Calin.Cenan@cs.utcluj.ro \\ ${ }^{2}$ Medical Pharmacy University Cluj-Napoca
}

\begin{abstract}
The paper presents the architecture of a distributed software system that manages patients' health records and assures remote and interactive access to medical services. In order to cover the complex relationships between different medical concepts (symptoms, diseases, treatment, medication, etc.) and also to include "intelligence" in the software system an ontology-based approach was adopted. This solution together with the adoption of some widely accepted medical standards (e.g. HL7, LOINC, etc.) assure interoperability and transparent data exchange between various medical applications. The paper presents a distributed healthcare system model in which patients, doctors and software components interact in order to assure better medical services. The key factor in the implementation of this model is the extensive use of the domain's ontology as the bases for database definition and medical data processing. In order to show the usefulness of the proposals as well as their effectiveness in electronic management of patient's health records an experimental implementation of the proposed methodology is in work in the filed of cardio-vascular diseases.
\end{abstract}

\section{Introduction}

All the modern concepts of medical services are centered on the healthcare consumer, the patient who receives medical attention or treatment. Recent developments in information technology and communication offer new opportunities in the implementation of high quality healthcare systems. These technologies assure the support for better medical data processing, more accurate diagnoses and easier access to medical services.

Today a major request imposed for medical systems is the integration of the different medical applications and services, regardless of their regional distribution, ownership or specific medical domains. A patient should access medical services in a uniform and transparent way anytime and anywhere. His/her medical records should travel seamlessly between medical entities (e.g. general practitioner, hospital, laboratory) as required by his/her treatment path. This can be achieved with the intensive use of standards and with the adoption of a generally accepted terminology.

Clearly an ontology-based approach is needed in order to represent entities, ideas, and events, along with their properties and relations, as a form of knowledge representation about the medical world [1]. Unlike many other disciplines, medicine has a long standing tradition in structuring its domain knowledge, e. g. disease taxonomies, medical procedures, anatomical terms and others, in a wide variety of medical terminologies, thesauri and classification systems.

A number of international organizations (e.g. HL7 consortium, ISO, CEN, etc.) are involved in a complex effort towards global classification and coding of concepts and terms used in medical domain. There are also a number of international research project focused on the development of new ITC models and methodologies for better medical information management.

This paper present our proposal for a healthcare information system dedicated for cardio-vascular diseases. Through the extension of the ontology the proposed system may be used also for other medical domains.

The rest of the article is organized as follows: The second chapter presents the current state of research in the domain and the third one presents the proposed distributed, patient-centric healthcare system model. The next parts of the paper are dedicated to the principles behind our ontological approach and the way we built our medical knowledge base. 


\section{Related Work}

Medical informatics standards are critical for design of terminologies, which are increasingly used to populate clinical databases. Medical classification, or medical coding, is the process of transforming descriptions of medical diagnoses and procedures into universal values. Some widely recognized medical coding standards used in our system are:

- Diagnosis-Related Group (DRG) - system to classify hospital cases

- International Classification of Diseases and related health problems, $10^{\text {th }}$ revision (ICD-10) [2]

- Logical Observation Identifiers Names and Codes (LOINC) - database and universal standard for identifying laboratory observations [3]

- National Drug Code (NDC) - directory of registered drug establishments provided by U.S. Food and Drug Administration (FDA) [4]

- Health Level Seven (HL7) provide standards for the exchange, of electronic health information [5]

The medical community has long been sensitive to the need of modeling its knowledge and of making its terminologies explicit. Therefore, there exist several terminological or ontological resources that model parts of the medical domain: controlled medical vocabularies such as the Systematized Nomenclature of Medicine, Clinical Terms (SNOMED CT) [6], GALEN [7], MENELAS [8], ONIONS library [9], or the highly complex UMLS [10] used to allow a standard, accurate exchange of data content between different systems and providers.

SNOMED, the Systematized Nomenclature of Medicine, is a standardized medical vocabulary that has been accepted internationally. Intended to completely and logically interrelate groupings of defined medical terms, SNOMED is a formalized, information-packed set of more than 300,000 coded medical terms.

UMLS, the Unified Medical Language System is an umbrella system which covers many medical thesauri and classifications. From a conceptual perspective, the UMLS can be divided into a Semantic Network (SN) which forms the upper ontology and consists of semantic types linked by semantic relations and a Meta-thesaurus which contains concepts assigned to one or more types. Given the size, the evolutionary diversity and inherent heterogeneity of this huge UMLS semantic network, there is no surprise that the lack of a formal semantic foundation leads to inconsistencies and circular definitions [11].

\section{Architecture of healthcare system}

In order to define the system's architecture a number of aspects must be taken into consideration. The first is the distributed nature of the medical data and services. Every entity involved in the medical system (general practitioners, hospitals, laboratories) should administer their one medical information using an appropriate application (server). But, a patient treatment may involve a number of entities, so their corresponding applications must exchange medical data concerning the patient. Automatic data transfer must be assured when a patient goes from his/her general practitioner to the laboratory or to the hospital and backwards.

Another important aspect taken into consideration is the possibility for a patient to access medical services from home, using a terminal (PC, PDA, intelligent phone). The system must allow off-line or on-line consultations and remote data acquisition from portable medical equipments.

The medical data security is another important issue. All the transfers must be done in accordance with the privacy rules accepted in medical systems.

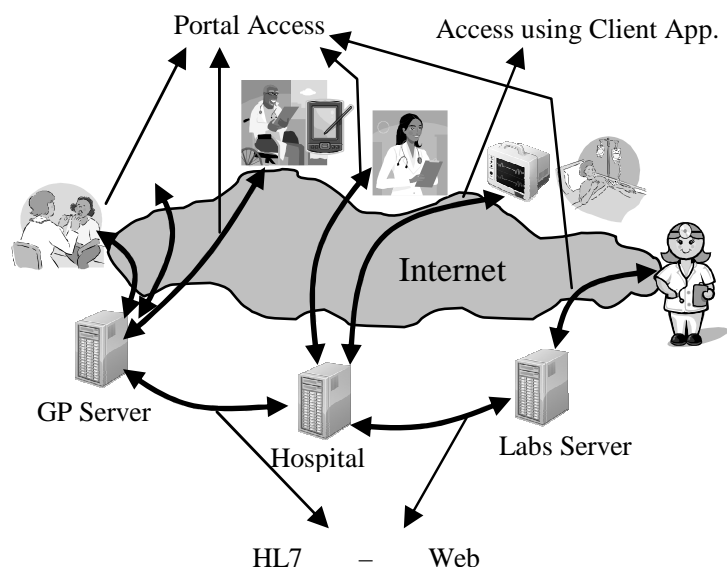

Fig1. General view of the Distributed system

Figure 1 shows the general view of the proposed system, in which the following elements can be identified:

- medical applications (servers) adapted for every type of medical entity (general practitioner office, hospital, laboratory application)

- main actors of the system: patients at home, general practitioners, specialists (in hospitals), laboratory analysts

- interactions between actors and servers and between servers

A medical application (server) has a multi-layer structure. Figure 2 shows the main software components organized on the following layers: medical 
knowledge database layer, access control layer, data processing layer and user and service interface layer

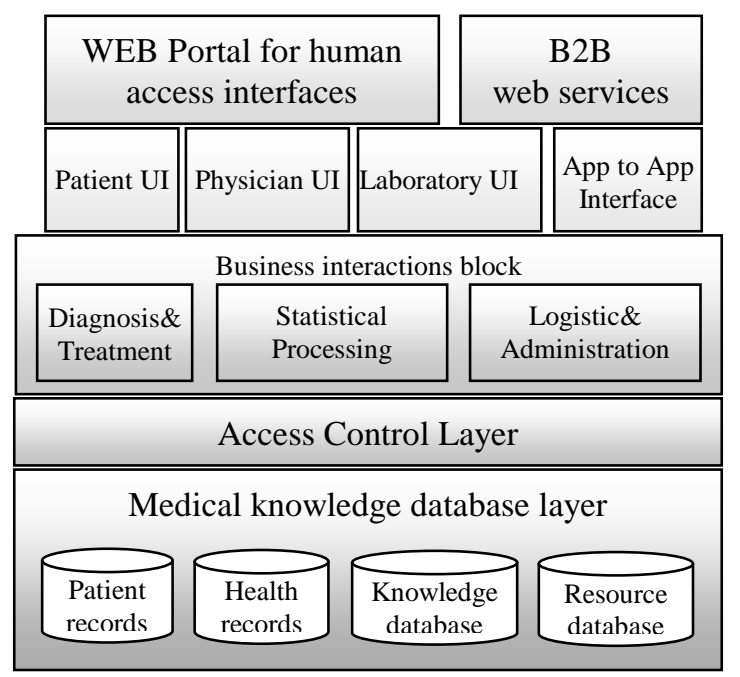

Fig2. Multi layer structure of the Distributed Healthcare System

The database is structured on 4 components: patient records, medical documents, knowledge base and resources database. The patient records part stores all the medical information concerning patients: demographic data (recorded once) and periodical observations (results of consults). The knowledge base preserves all the concepts and relations defined in the domain's ontology and it is the bases for medical reasoning and decision. The proposed ontology will support applications both individually and, more importantly, within an environment of heterogeneous inter-working clinical information systems. The resources database is used to administer financial and medical resources (e.g. treatment costs, drugs, equipments' usage, etc.) The medical documents part preserves all the official documents generated during the healthcare process.

The access control layer is responsible for the authorization of users for different operations: recording, viewing medical data, modifying records, etc. The business interaction block is managing the interactions between the actors and the system during a medical procedure in accordance with rules in the ontology (based on treatment plans). The diagnosis and treatment module is the decision support unit for the medical diagnoses. This module generates suggestions concerning treatments based on the patient's medical data and rules specified in the ontology. The statistical processing module contains a set of predefined procedures that allow statistical evaluations on the medical data repository. The administration module is used to keep track of the usage of resources, cost and will export data to other resource management systems.

\section{Ontology for the Cardiology Domain}

In our approach the ontology has the central role of knowledge representation. It contains domain concepts and knowledge about patient characteristics.

The main part of the ontology implemented in our project is inspired by a knowledge base that should assist in management of heart failure patients. The current version of the constructed heart failure ontology is presented in [12] and it is available at http://lis.irb.hr/heartfaid/ontology/. The design of the heart failure ontology has started from the terms defined in [13]. In order to connect the ontology concepts with the terms defined in UMLS appropriate references were introduced for every concept.

We built the medical ontology encoded in an internal format of a database, without a representation readable by a human user. All the ontology's elements were properly translated in our database. So we have tables in our database to store concepts, instances of concepts, concept hierarchy and relationships between different concepts and instances, synonyms and UMSL synonyms. The slots from ontology are a little bit difficult to store in the database so we need many tables to store these properties: tables to store slot's characteristics like name, tables to store possible associations, and tables to store the actual values of these slot. According to this we have for example tables to store the fact that "Indicated" is the name of a slot connecting instances and also that this slot is a valid property only for instances of concepts "Patient characteristics", "Testing", "CHF risks", "Classification" and "Treatment". We can store the fact that for "Felodipine" (which is an instance of "Calcium antagonist" and further an instance of the super class "Heart failure medication group") this slot is related to instances like "Diastolic hypertension", "Unstable angina" and "Systolic hypertension" which are instances of concept "Patient characteristics". For example the instances "Diastolic hypertension" and "Systolic hypertension" of concept "Hypertension" are "Patient characteristics" via sub classes "Diagnosis", "Cardiovascular system related", "Artery and blood disorder" and "Blood pressure disorder".

Medical plans have the goal of indicating the decisions and tasks most appropriate for optimizing health outcomes and controlling costs. They are built either from loose indications of a preferred set of choices or from normative rules requiring more or less strict adherence. 
One of the main theoretical contributions of our work was the decision to represent in our ontology the medical plans using Process Specification Language, PSL [14], merging the PSL ontology and the Heart Failure ontology in our healthcare system.

The Process Specification Language (PSL) is developed by the American NIST, National Institute of Standards and Technology, and defines a neutral representation for processes that supports automated reasoning. The initial use of this ontology was for manufacturing processes.

There are four kinds of entities required for reasoning about processes: activities, activity occurrences, timepoints, and objects.

- (activity ?a) - activities can be considered to be reusable behaviors within the domain

- (activity_occurrence ?occ) - an activity occurrence is associated with a unique activity and begins and ends at specific points in time.

- (object ?x), (participates_in ?x ?occ ?t) - an object is anything that is not a time point, nor an activity nor an activity occurrence. Intuitively, an object is a concrete or abstract thing that can participate in an activity.

An example taken from our approach can state that

(take_care Patient1 Doctor1)

is an instance of the class of Treatment activities:

(Treatment_activities (take_care Patient1 Doctor1))

There may be multiple distinct occurrences of this instance which consequently will be recorded in many episodes from the medical life of the patient:

(occurrence_of Occ1 (take_care Patient1 Doctor1))

(occurrence_of Occ2 (take_care Patient1 Doctor1))

$(=($ beginof $\mathrm{Occ} 1) \mathrm{t} 1),(=($ endof Occ 1$) \mathrm{t} 2)$

$(=($ beginof Occ 2$) \mathrm{t} 5),(=($ endof Occ 2$) \mathrm{t} 8)$

\section{Conclusions}

Our research dealt with new ways of delivering health care in a coordinated approach. We propose distributes architecture for efficient management of patients' health record. The application assures interactive and real time data exchange between the main actors of the medical system.

Using ontology and widely accepted medical coding standards the proposed solution solves the interoperability issues between medical entities. Rules and relations embedded in the medical knowledge based offer support for a better medical decision and in these ways assure higher quality for medical services. Also the system assures remote access and patient's monitoring reducing time and cost needed for medical assistance.
Acknowledgment: Work on this paper is supported under the auspices of CARDIONET, national research grant funded by ANCS, CNMP-PC, 11-01/2007.

\section{References}

[1] A. L. Rector, W. D. Solomon, W. A. Nowlan, T. W. Rush, "A terminology server for medical language and medical information systems", Methods of Information in Medicine, Vol. 34, pp. 147-157, North Holland, 1995

[2] International Statistical Classification of Diseases and Health Related Problems (The) ICD-10 Second Edition, World Health Organization, 2004.

[3] M. Stark, "A look at LOINC - The Established Standard for Lab Data Gains Visibility as Data Exchange Increases", Journal of American Health Information Management Association, 77(7):52, 54-5; 57-8, 2006.

[4] U.S. Food and Drug Administration (FDA), The National Drug Code Directory, http://www.fda.gov/cder/ndc/, 2008.

[5] Health Level 7 (HL7) http://www.hl7.org, 2008.

[6] K. Spackman, K. Campbell, R. Côté, "SNOMED RT: a reference terminology for health care", Proc. of the 1997 AMIA Symposium, pp. 25-29, Nashville, 1997.

[7] A. L. Rector, J. Rogers, P. Pole, "The GALEN high level ontology", Proc. of Medical Informatics in Europe, pp. 174-178, 1996.

[8] P. Zweigenbaum, Consortium Menelas, "Menelas: Coding and information retrieval from natural language patient discharge summaries", Advances in Health Telematics, IOS Press, Amsterdam, pp. 82-89, 1995.

[9] A. Gangemi, D. M. Pisanelli, G. Steve, "An overview of the ONIONS project: applying ontologies to the integration of medical terminologies", Data \& Knowledge Engineering, Vol. 31, pp. 183-220, 1999.

[10] D. M. Pisanelli, A. Gangemi, G. Steve, "A Medical ontology library that integrates the UMLS MetathesaurusTM", Lecture Notes In Computer Science; Vol. 1620 Proc. of Joint European Conf. on Artificial Intelligence in Medicine and Medical Decision Making, pp. 239-248, 1999.

[11] K. E. Campbell, D. E. Oliver, E. H. Shortliffe, "The Unified Medical Language System: toward a collaborative approach for solving terminologic problems", Journal of American Medical Informatics Association, Vol. 5 Jan-Feb, pp. 12-6, 1998.

[12] A. Jovic, M. Prcela, D. Gamberger, "Ontologies in Medical Knowledge Representation”, Proc. of Int. Conf. Information Technology Interfaces, pp: 535 - 540, 2007.

[13] European Society of Cardiology - Task Force, "Guidelines for diagnosis and treatment of the chronic heart failure", http://www.escardio.org, 2005.

[14] J.J. Michel, A.F. Cutting-Decelle, "The Process Specification Language," International Standards Organization ISO TC184/SC5 Meeting, Paris, April 2004. 